\title{
Fatores associados à fragilidade em idosos acompanhados na Atenção Primária à Saúde
}

\author{
Factors associated with frailty in elderly patients followed up in primary health care
}

Factores asociados a la fragilidad en ancianos acompañados en la Atención Primaria de Salud

Pryscila Ravene Carvalho Oliveira ${ }^{1}$ (1) Vitória Eduarda Silva Rodrigues ${ }^{1}$ (1) Ana Karoline Lima de Oliveira ${ }^{1}$ (1) Francisco Gerlai Lima Oliveira ${ }^{1}$ (1) Gabriela Araújo Rocha ${ }^{1}$ (1) Ana Larissa Gomes Machado ${ }^{1}$ (D)

1. Universidade Federal do Piauí. Picos, $\mathrm{PI}$, Brasil.
Autor correspondente:

Pryscila Ravene Carvalho Oliveira.

E-mail: prys_carvalho@outlook.com

Recebido em 27/08/2020.

Aprovado em 11/02/2021.

DOI:https://doi.org/10.1590/2177-9465-EAN-2020-0355

\section{Resumo}

Objetivo: Verificar a associação entre os marcadores de fragilidade e as características sociodemográficas e clínicas em idosos na Atenção Primária à Saúde. Método: Pesquisa transversal, realizada com 356 idosos cadastrados em unidades de saúde da família, no Nordeste do Brasil. Os instrumentos de coleta utilizados foram o Índice de Vulnerabilidade Clínico-Funcional e outro de perfil socioeconômico e de saúde. Analisaram-se os dados pela estatística inferencial, utilizando o teste do Qui-quadrado, com nível de significância 0,05. Resultados: A média de idade foi de 72,85 anos $( \pm 8,965) ; 63,8 \%$ eram do sexo feminino, 39\% apresentaram risco de fragilização e $22,5 \%$ demonstraram fragilidade. Houve associação entre fragilidade, sexo, faixa etária estado civil, escolaridade, renda, presença de doença cardíaca e hipertensão. Conclusões e implicações para a prática: Os marcadores de fragilidade nos idosos evidenciaram associação entre as características sociodemográficas e clínicas, indicando a necessidade de rastreio precoce no nível primário de atenção à saúde.

Palavras-chave: Saúde do Idoso; Fragilidade; Atenção Primária à Saúde; Vulnerabilidade em Saúde; Qualidade de Vida.

\section{Abstract}

Objective: to verify the association between frailty markers and sociodemographic and clinical characteristics in elderly people in Primary Health Care. Method: cross-sectional research, carried out with 356 elderly people registered in family health units in the Northeast of Brazil. The collection instruments used were the Index of Clinical-Functional Vulnerability and another of socioeconomic and health profile. The data were analyzed by inferential statistics, using the chi-square test, with a significance level of 0.05 . Results: the mean age was 72.85 years $( \pm 8.965) ; 63.8 \%$ were female, $39 \%$ were at risk of frailty and $22.5 \%$ were frail. There was an association between frailty, gender, age group, marital status, education, income, presence of heart disease and hypertension. Conclusions and implications for practice: the markers of frailty in the elderly showed an association between sociodemographic and clinical characteristics, indicating the need for early screening at the primary health care level.

Keywords: Health of the Elderly; Frailty. Primary Health Care; Health Vulnerability; Quality of Life.

\section{REsumen}

Objetivo: Verificar la asociación entre los marcadores de fragilidad y las características sociodemográficas y clínicas en ancianos en Atención Primaria de Salud. Método: Investigación transversal realizada con 356 ancianos registrados en unidades de salud familiar en Nordeste de Brasil. Los instrumentos de recolección utilizados fueron el Índice de Vulnerabilidad Clínico-Funcional y otro de perfil socioeconómico y de salud. Los datos se analizaron mediante estadística inferencial utilizando la prueba de Chicuadrado, con nivel de significancia de 0.05 . Resultados: La edad media fue de 72,85 años $( \pm 8,965)$, el $63,8 \%$ eran mujeres el $39 \%$ tenían riesgo de fragilidad y el 22,5\% eran frágiles. Hubo asociación entre fragilidad, sexo, edad, estado civil, educación, ingresos, presencia de cardiopatía e hipertensión. Conclusiones e implicaciones para la práctica: Los marcadores de fragilidad en ancianos se asociaron con características sociodemográficas y clínicas, lo que indica la necesidad de rastreo precoz en el nivel primario de atención a la salud.

Palabras clave: Salud del Anciano; Fragilidad; Atención Primaria de salud; Vulnerabilidad en Salud; Calidad de Vida. 


\section{INTRODUÇÃO}

O processo de envelhecimento tem caráter progressivo e plurifacetado, no qual acontecem alterações físicas e mentais que, na sua maioria, geram consequente diminuição gradual da capacidade funcional. ${ }^{1}$ Essas mudanças estão estreitamente relacionadas à fragilidade, tornando os indivíduos mais vulneráveis a danos. ${ }^{2}$

Uma eventualidade que preocupa gestores e profissionais que trabalham com idosos é a fragilidade, considerada uma síndrome multidimensional que envolve a interação de fatores biológicos, psicológicos e sociais. O indivíduo torna-se susceptível à desregulação dos sistemas neuroendócrino e imunológico, e, consequentemente, sua capacidade em resistir aos eventos estressores em saúde é comprovadamente reduzida. ${ }^{3} \mathrm{~A}$ fragilidade está relacionada ao maior risco de ocorrência de desfechos adversos, como declínio na capacidade funcional, quedas, delírio, institucionalização, hospitalização e morte. ${ }^{4}$

O fenótipo de fragilidade é composto pelos seguintes fatores: perda de peso não intencional; presença de fadiga autorreferida; redução da força de preensão; lentidão na velocidade da marcha lenta; e pouca prática de atividade física. Assim, a perda de peso sem motivo aparente, fraqueza, diminuição da força muscular, dificuldades de caminhar e hábitos sedentários podem ser fatores preditores e de risco para desenvolver a síndrome de fragilidade. ${ }^{5}$

Dessa forma, faz-se necessário reconhecer a sintomatologia de toda a dinâmica do comprometimento funcional, visto que a fragilidade pode estar instalada e impactando negativamente a vida do idoso, de seus cuidadores e familiares. Além disso, é preciso compreender que há sinergia de fatores intrínsecos e extrínsecos, observando, assim, que a idade avançada, sexo feminino, pobreza, baixa escolaridade, tabagismo, baixo índice de massa corporal e presença de doenças crônicas são fatores preditivos de fragilidade. ${ }^{6}$

A detecção da Síndrome da Fragilidade Clínico Funcional (SFCF) pode ser realizada por meio da observação de fatores de risco e de instrumentos de avaliação apropriados, por exemplo, o Índice de Vulnerabilidade Clínico-Funcional-20 (IVCF-20), o qual possui linguagem simples e acessível, pode ser utilizado por pessoas leigas e profissionais da saúde, e padroniza a classificação da fragilidade ao identificá-la de maneira rápida. O IVCF-20 representa um avanço nacional, pois é o primeiro instrumento brasileiro para identificação do idoso frágil; e está classificado entre os quatro melhores do mundo, sendo referência em geriatria ao realizar a triagem de fragilidade. ${ }^{7}$

Na contemporaneidade, poucos são os estudos que abordam os prognósticos de fragilidade com indicadores simples e de fácil aplicação no momento da triagem. ${ }^{8}$ Nesse sentido, considerase relevante a utilização de instrumentos de rápido rastreio da SFCF, na Atenção Primária à Saúde (APS), subsidiando ações da equipe de enfermagem para melhor planejamento dos cuidados aos idosos.

A equipe de enfermagem atuante na APS desempenha uma importante função como cuidadora dos idosos, e a identificação precoce de fatores associados à SFCF na população contribui sobremaneira para que os cuidados gerontológicos sejam efetivos e precoces, retardando a perda da funcionalidade. ${ }^{9}$

Nesse cenário, evidencia-se a relevância da identificação e discussão acerca dos marcadores de fragilidade na APS, pois favorece a avaliação multidimensional do idoso e, a partir do seu aprofundamento, fornece subsídios para implementar políticas públicas e planejar ações estratégicas de saúde que impactem positivamente a vida do idoso, de sua família e comunidade, justificando-se, assim, a realização deste estudo.

Dessarte, objetivou-se verificar a presença de marcadores de fragilidade e sua relação com as características sociodemográficas e clínicas em idosos na atenção primária à saúde.

\section{MÉTODO}

Estudo quantitativo, observacional e transversal, realizado em Unidades de Saúde da Família (USF) da cidade de Picos-PI, no Nordeste do Brasil. O município conta com 36 Equipes de Saúde da Família (ESF), sendo 25 localizadas na zona urbana e 11 na zona rural.

A população do estudo foi composta por 4.895 idosos acompanhados pelas ESF do município, os quais atenderam aos critérios de inclusão: possuir 60 anos ou mais de idade, estar cadastrado na USF da zona urbana, apresentar comunicação verbal preservada e compreensão das instruções repassadas.

Como critério de exclusão, adotou-se a presença de déficits cognitivos, identificados pelo baixo desempenho no Mini-Exame do Estado Mental (MEEM). O uso desse teste é importante para avaliar se o idoso apresenta déficit cognitivo, ao identificar indivíduos com risco de desenvolver uma síndrome demencial. O MEEM é composto por questões agrupadas em sete categorias: orientação para o tempo, memória imediata, atenção e cálculo, evocação, lembrança de palavras, linguagem, e capacidade construtiva visual. ${ }^{10}$

Para o cálculo amostral, foi utilizado como desfecho um percentual de $50 \%$ ( $P=50 \%$ e $Q=50 \%)$, haja vista que esse valor proporciona um tamanho máximo de amostra, sendo fixados o nível de significância $(\alpha=0,05)$, o erro tolerável de amostragem de $5 \%$, e a variável reduzida $(Z=1,96)$. A amostra foi calculada por fórmula para população finita, composta por 356 participantes, e estratificada com divisão proporcional entre todas as USF da zona urbana, conforme o Quadro 1:

A coleta de dados ocorreu no período de setembro de 2018 a junho de 2019, sendo realizada tanto nas UBS quanto nos domicílios dos idosos, pois dependia da disponibilidade do participante da pesquisa. Utilizaram-se dois instrumentos: o IVCF-20 (Índice de Vulnerabilidade Clínico-Funcional-20), o qual tem caráter multidimensional e alta confiabilidade e se propõe a avaliar os principais marcadores de fragilidade do idoso; e um formulário sobre os dados sociodemográficos e condições de saúde.

OIVCF-20 foi criado com base na Avaliação Geriátrica Ampla (AGA) e validado no ano de 2014, como um instrumento de triagem interdisciplinar, que contempla aspectos multidimensionais da condição de saúde dos idosos. É composto por 20 questões 
Quadro 1. Estratificação da amostra nas Unidades Básicas de Saúde da zona urbana. Picos, PI, Brasil, 2019

\begin{tabular}{|c|c|c|}
\hline UBS & POPULAÇÃO & AMOSTRA \\
\hline Unidade A & 222 & 16 \\
\hline Unidade B & 80 & 6 \\
\hline Unidade C & 92 & 8 \\
\hline Unidade D & 66 & 4 \\
\hline Unidade E & 223 & 16 \\
\hline Unidade F & 272 & 20 \\
\hline Unidade G & 145 & 11 \\
\hline Unidade H & 187 & 14 \\
\hline Unidade I & 296 & 22 \\
\hline Unidade J & 333 & 25 \\
\hline Unidade K & 178 & 12 \\
\hline Unidade L & 190 & 15 \\
\hline Unidade M & 238 & 18 \\
\hline Unidade N & 240 & 19 \\
\hline Unidade 0 & 291 & 20 \\
\hline Unidade P & 230 & 16 \\
\hline Unidade Q & 78 & 5 \\
\hline Unidade R & 269 & 19 \\
\hline Unidade S & 310 & 22 \\
\hline Unidade T & 193 & 14 \\
\hline Unidade U & 55 & 4 \\
\hline Unidade V & 84 & 7 \\
\hline Unidade X & 74 & 6 \\
\hline Unidade W & 143 & 10 \\
\hline Unidade $Y$ & 187 & 13 \\
\hline Unidade Z & 219 & 14 \\
\hline TOTAL & 4895 & 356 \\
\hline
\end{tabular}

Fonte: dados da pesquisa.

distribuídas em 8 seções, incluindo idade (1 questão), autopercepção da saúde (1 questão), atividades de vida diária (4 questões), cognição (3 questões), humor (2 questões), mobilidade (6 questões), comunicação (2 questões) e comorbidades múltiplas ou internação recente (1 questão). ${ }^{11}$

Quanto maior a pontuação do idoso no IVCF-20 - cujo total é 40 de pontos -, pior será sua condição clínico-funcional, estratificando-o em: robusto (0 a 6 pontos), que apresenta boa reserva homeostática, independência e autonomia e sem nenhuma incapacidade funcional; risco de fragilização (7 a 14 pontos), que, apesar de gerenciar sua vida com independência e autonomia, já apresenta limitações funcionais; e, por último, frágil (15 ou mais pontos), que possui declínio funcional e incapacidades únicas ou múltiplas, tornando-se incapaz de gerenciar a própria vida. ${ }^{12}$
As variáveis sociodemográficas e de saúde selecionadas foram: idade, escolaridade, estado civil, renda, presença de doenças e Índice de Massa Corporal (IMC).

Os resultados foram tabulados no programa Statistic Package for Social Science versão 20.0, e realizou-se o Teste de normalidade de Kolmogorov-Smirnov na distribuição das variáveis analisadas. Quanto à análise dos dados, optou-se pela estatística inferencial, utilizando-se o teste de Qui-quadrado para o estudo das associações entre as variáveis categóricas, com nível de significância 0,05.

A pesquisa obteve aprovação ética pelo Comitê de Ética em Pesquisa, com Parecer $n . \stackrel{0}{2}$ 2.389.117. Em seguida, todos os participantes foram informados sobre os seus objetivos e, após sua anuência, assinaram o Termo de Consentimento Livre e Esclarecido (TCLE).

\section{RESULTADOS}

O estudo incluiu 356 idosos, com média de idade de 72,85 anos $( \pm 8,965)$, sendo $63,8 \%$ do sexo feminino. Em relação ao grau de fragilidade, conforme a classificação proposta pelo IVCF20, observou-se que: 139 (39\%) possuíam risco de fragilização, $137(38,5 \%)$ eram robustos e $80(22,5 \%)$ frágeis.

Os dados acerca do perfil sociodemográfico dos idosos, assim como o perfil de classificação da fragilidade, foram descritos na Tabela 1. Houve diferença significativa para todas as variáveis analisadas.

A Tabela 2 apresenta as condições de saúde dos idosos, segundo os níveis de fragilidade. Houve diferença significativa entre as variáveis doença cardíaca e hipertensão - 14,3\% e $64,3 \%$, respectivamente -, as quais foram relatadas com maior frequência pelos idosos. Com relação ao IMC, chama a atenção o percentual de idosos com sobrepeso $(54,0 \%)$, representando $46,3 \%$ dos frágeis.

$\mathrm{Na}$ Tabela 3, destacam-se os marcadores de fragilidade apresentados pelos idosos.

Mediante a análise dos marcadores de fragilidade, notou-se não haver frequências elevadas nos diferentes aspectos avaliados. Portanto, merecem destaque a autopercepção da saúde relatada como ruim ou péssima por $44,1 \%$ dos participantes, e aos percentuais de idosos com dependência para AVD instrumental (23,6\%), quando comparados aos idosos com dependência em AVD básica $(4,8 \%){ }^{13}$

Outro dado com destaque foi o humor, pois $44,7 \%$ dos idosos relataram sentir desânimo, tristeza ou desesperança nos últimos meses, e 26,7\% perderam o interesse em atividades anteriormente prazerosas.

No que concerne à mobilidade, houve predominância de respostas referentes à boa mobilidade em todos os itens avaliados. Porém, deve-se considerar que, acerca da capacidade aeróbica/muscular dos idosos, a circunferência da panturrilha menor que $31 \mathrm{~cm}$ esteve presente em $23,6 \%$ dos participantes, demonstrando a existência de sarcopenia entre eles. No que tange às comorbidades múltiplas, a polifarmácia foi o item mais apontado na amostra estudada $(15,7 \%) .{ }^{13}$ 
Tabela 1. Variáveis sociodemográficas e níveis de fragilidade (n=356). Picos, PI, Brasil, 2019

\begin{tabular}{|c|c|c|c|c|c|}
\hline \multirow{2}{*}{ Variáveis } & Robusto & Risco de fragilização & Frágil & Total & \multirow{2}{*}{ p-valor* } \\
\hline & $n(\%)$ & $n(\%)$ & $n(\%)$ & n (\%) & \\
\hline Sexo & & & & & 0,046 \\
\hline Feminino & $80(58,4)$ & $87(62,6)$ & $60(75,0)$ & $227(63,8)$ & \\
\hline Masculino & $57(41,6)$ & $52(37,4)$ & $20(25,0)$ & $129(36,2)$ & \\
\hline Faixa etária & & & & & 0,001 \\
\hline $60-74$ & $110(80,3)$ & $86(61,9)$ & $32(40,0)$ & $228(64,0)$ & \\
\hline $75-84$ & $27(19,7)$ & $41(29,5)$ & $25(31,2)$ & $93(26,1)$ & \\
\hline$\geq 85$ & $0(0)$ & $12(8,6)$ & $23(28,8)$ & $35(9,8 \%)$ & \\
\hline Estado civil & & & & & 0,001 \\
\hline União estável ou casado & $97(70,8)$ & $72(51,8)$ & $34(42,5)$ & $203(57,0)$ & \\
\hline Viúvo & $25(18,2)$ & $51(36,7)$ & $33(41,3)$ & $109(30,6)$ & \\
\hline Solteiro & $15(10,9)$ & $16(11,5)$ & $13(16,3)$ & $44(12,4)$ & \\
\hline Escolaridade & & & & & 0,001 \\
\hline Não letrado & $50(36,5)$ & $55(39,6)$ & $35(43,8)$ & $140(39,3)$ & \\
\hline Até 8 anos de estudo & $50(36,5)$ & $66(47,5)$ & $40(50,0)$ & $156(43,8)$ & \\
\hline Mais de 8 anos de estudo & $37(27,0)$ & $18(12,9)$ & $5(6,3)$ & $60(16,9)$ & \\
\hline Renda & & & & & 0,047 \\
\hline$\geq 1$ salário mínimo & $107(78,1 \%)$ & $122(87,8)$ & $61(76,3)$ & $290(81,5)$ & \\
\hline < 1 salário mínimo & $30(21,9)$ & $17(12,2)$ & $19(23,8)$ & $66(18,5)$ & \\
\hline
\end{tabular}

Fonte: dados da pesquisa. * Teste de associação qui-quadrado $(p<0,05)$.

\section{DISCUSSÃO}

Considerando as variáveis sociodemográficas, foi possível mapear uma população constituída predominantemente pelo sexo feminino - um achado já esperado e consoante com várias literaturas. ${ }^{14,15} \mathrm{~A}$ predominância de fragilidade entre as mulheres idosas é semelhante a outra pesquisa de base populacional, ${ }^{16}$ em que 11.015 homens e mulheres com mais de 60 anos de idade foram acompanhados durante dois anos e avaliados quanto ao fenótipo de fragilidade. $O$ estudo concluiu que houve prevalência específica de fragilidade no sexo feminino, uma vez que as mulheres idosas têm quase o dobro de probabilidade de serem frágeis do que os homens ( $16,4 \%$ vs. $8,6 \%$ ), e também são mais propensas ao risco de fragilização. ${ }^{16}$

As mulheres apresentam maior longevidade em relação aos homens; em contrapartida, demonstram qualidade de vida inferior. As diferenças de gênero interferem no alcance de oportunidades sociais - como o acesso à saúde - e estão presentes em todo o ciclo vital. ${ }^{17} \mathrm{~A}$ desigualdade ocorre tanto por serem mulheres, quanto por serem idosas, fato que as coloca em uma situação de dupla vulnerabilidade e potencializa a ocorrência de agravos à saúde. Tal constatação sugere a necessidade de uma atenção mais centrada para esse público em questão, objetivando um atendimento com equidade. ${ }^{18}$

Os resultados confirmam maior frequência de idosos com fragilidade, e risco de fragilidade devido ao aumento da idade, sendo possível observar a redução de idosos robustos o aumento da idade. O risco de tornar-se frágil é mais presente a partir dos 80 anos, com risco 1,24 vezes maior se comparado à faixa entre 65 e 79 anos. ${ }^{19,20}$ Porém, outros fatores também são preponderantes para o aparecimento da síndrome da fragilidade, como uma ou mais internações, aumentando em 4,3 a prevalência de ocorrer fragilidade. Ademais, a polipatologia e a polifarmácia são outras causas sinérgicas e contribuintes para a Síndrome da Fragilidade Clínico-Funcional. ${ }^{21}$ Percebe-se, pois, que o desenvolvimento da fragilidade não está unicamente relacionado à idade ou ao processo de envelhecimento. 
Tabela 2. Características de saúde e níveis de fragilidade (n=356). Picos, PI, Brasil, 2019

\begin{tabular}{|c|c|c|c|c|c|}
\hline $\begin{array}{c}\text { Variáveis } \\
\text { n (\%) }\end{array}$ & $\begin{array}{c}\text { Robusto } \\
\text { n (\%) }\end{array}$ & $\begin{array}{c}\text { Risco de fragilização } \\
\mathrm{n}(\%) \\
\end{array}$ & $\begin{array}{l}\text { Frágil } \\
\mathrm{n}(\%) \\
\end{array}$ & Total & p-valor* \\
\hline Doença cardíaca & & & & & 0,003 \\
\hline Sim & $11(8,0)$ & $20(14,4)$ & $20(25,0)$ & $51(14,3)$ & \\
\hline Não & $126(92,0)$ & $119(85,6)$ & $60(75,0)$ & $305(85,7)$ & \\
\hline Hipertensão & & & & & 0,040 \\
\hline Sim & $77(56,2)$ & $97(69,8)$ & $55(68,8)$ & $229(64,3)$ & \\
\hline Não & $60(43,8)$ & $42(30,2)$ & $25(31,3)$ & $127(35,7)$ & \\
\hline Diabetes & & & & & 0,249 \\
\hline Sim & $28(20,4)$ & $37(26,6)$ & $24(30,0)$ & $89(25,0)$ & \\
\hline Não & $109(79,6)$ & $102(73,4)$ & $56(70,0)$ & $267(75,0)$ & \\
\hline IMC** & & & & & 0,133 \\
\hline Baixo Peso & $5(3,6)$ & $6(4,3)$ & $8(10,0)$ & $19(5,3)$ & \\
\hline Adequado & $50(36,5)$ & $60(43,2)$ & $35(43,7)$ & $145(40,7)$ & \\
\hline Sobrepeso & $82(59,9)$ & $73(52,5)$ & $37(46,3)$ & $192(54,0)$ & \\
\hline
\end{tabular}

Fonte: dados da pesquisa. ${ }^{*}$ Teste de associação qui-quadrado $(p<0,05) .{ }^{* *}$ IMC: Índice de massa corporal.

A baixa escolaridade apresentada pelos idosos com risco de fragilidade e frágeis impacta negativamente na busca pela assistência e nas práticas de autocuidado. Somados a isso, os poucos anos de estudos ou a falta de letramento sugerem uma situação socioeconômica desfavorável, tendo como consequência desigualdades sociais. ${ }^{22}$ Idosos que possuem baixa escolaridade procuram com menos frequência os serviços de saúde, por apresentarem pouco ou nenhum conhecimento acerca da necessidade de acesso aos serviços. Além disso, também está fortemente relacionado à incapacidade funcional, comprometimento cognitivo e risco de fragilidade em idosos. ${ }^{23}$ Isso fomenta a reflexão sobre os processos assistenciais e de gerência, na busca por um atendimento de qualidade e específico para as demandas individuais na APS.

Embora a presença de doenças crônicas não seja acompanhada de fragilidade, ela predispõe o idoso ao aumento da vulnerabilidade clínico-funcional. ${ }^{24}$ Tal constatação foi demonstrada em um estudo, no qual o aparecimento de doenças crônicas esteve associado ao avanço da idade, e a prevalência do diabetes associou-se à dependência para a realização de tarefas. ${ }^{22}$

Um estudo americano mostrou que as doenças cardíacas estão associadas ao envelhecimento biológico e, como resultado, síndromes geriátricas como a fragilidade têm maior probabilidade de se apresentar. ${ }^{25}$ Ademais, uma metanálise demonstrou que a fragilidade afeta quase um em cada dois pacientes com IC. Outro estudo evidenciou que os pacientes com idades mais avançadas, ${ }^{26,27}$ propensos a diversas condições crônicas como cardiopatias e hipertensão arterial sistêmica - são mais propensos ao diagnóstico de fragilidade.
A síndrome de fragilidade resulta de múltiplas deficiências em diferentes órgãos e é caracterizada por reservas fisiológicas reduzidas e maior vulnerabilidade a estressores. Além disso, a doença cardiovascular é um problema comum na população idosa. Evidências demonstram que pacientes frágeis com doenças cardiovasculares apresentam pior prognóstico que pacientes não frágeis, e que a fragilidade é um fator de risco para insuficiência cardíaca incidente entre idosos. ${ }^{28}$

Embora a hipertensão arterial não tenha se apresentado como uma das doenças de maior associação com a fragilidade, deve ser levada em consideração no grupo de morbidades mais relacionadas à fragilidade, devido à sua alta prevalência na população idosa, priorizando seu rastreio entre os idosos. ${ }^{29}$

O Índice de Massa Corporal (IMC) apresentou diferenças significativas quanto à fragilidade, com a prevalência de sobrepeso entre os idosos frágeis e em risco de fragilização. Do mesmo modo, outro estudo transversal sobre a síndrome de fragilidade em idosos concluiu que os padrões musculares nos idosos em risco de fragilização e frágeis são inferiores, e as medidas de IMC e de centralização de gordura, mais elevadas com a progressão da síndrome. ${ }^{30}$

A predominância de sobrepeso em idosos frágeis e em risco de fragilização contrasta com os resultados de outro estudo brasileiro, relacionou baixos valores de IMC à fragilidade, ${ }^{8}$ demonstrou que indivíduos com valores de IMC abaixo de 23,4kg/ $\mathrm{m}^{2}$ possuem maior probabilidade de serem frágeis. Nesse sentido, o sobrepeso não esteve associado à fragilidade.

O estado nutricional do idoso acelera ou retarda o aparecimento da sua fragilidade. ${ }^{30}$ Outrossim, o excesso de peso corporal pode contribuir para o desenvolvimento de comorbidades durante o 
Tabela 3. Frequência dos marcadores de fragilidade ( $n=356)$. Picos, PI, Brasil, 2019

\begin{tabular}{|c|c|c|}
\hline Marcadores de Fragilidade & $\mathrm{n}$ & $\%$ \\
\hline \multicolumn{3}{|l|}{ Idade } \\
\hline $60-74$ & 228 & 64,0 \\
\hline $75-84$ & 93 & 26,1 \\
\hline$\geq 85$ & 35 & 9,8 \\
\hline \multicolumn{3}{|l|}{ Autopercepção da saúde } \\
\hline Excelente/Muito Boa/Boa & 199 & 55,9 \\
\hline Regular/Ruim & 157 & 44,1 \\
\hline \multicolumn{3}{|l|}{ Atividades de Vida Diária (AVD) } \\
\hline \multicolumn{3}{|l|}{ AVD Instrumental } \\
\hline \multicolumn{3}{|l|}{ Deixou de fazer compras } \\
\hline Sim & 82 & 23,0 \\
\hline Não & 274 & 77.0 \\
\hline \multicolumn{3}{|l|}{ Deixou de controlar seu dinheiro } \\
\hline Sim & 84 & 23,6 \\
\hline Não & 272 & 76,4 \\
\hline \multicolumn{3}{|c|}{ Deixou de realizar pequenos trabalhos domésticos } \\
\hline Sim & 58 & 16,3 \\
\hline Não & 298 & 83,7 \\
\hline \multicolumn{3}{|l|}{ AVD Básica } \\
\hline \multicolumn{3}{|l|}{ Deixou de tomar banho sozinho } \\
\hline Sim & 17 & 4,8 \\
\hline Não & 339 & 95,2 \\
\hline \multicolumn{3}{|c|}{ Cognição } \\
\hline \multicolumn{3}{|c|}{ Algum familiar falou que você está ficando esquecido } \\
\hline Sim & 137 & 38,5 \\
\hline Não & 219 & 61,5 \\
\hline \multicolumn{3}{|l|}{ Este esquecimento está piorando } \\
\hline Sim & 82 & 23,0 \\
\hline Não & 274 & 77,0 \\
\hline \multicolumn{3}{|c|}{ Esquecimento impede a realização de alguma atividade } \\
\hline Sim & 64 & 18,0 \\
\hline Não & 292 & 82,0 \\
\hline \multicolumn{3}{|l|}{ Humor } \\
\hline \multicolumn{3}{|l|}{ Desânimo, tristeza ou desesperança } \\
\hline Sim & 159 & 44,7 \\
\hline Não & 197 & 55,3 \\
\hline \multicolumn{3}{|c|}{ Perdeu o interesse/prazer em atividades anteriormente prazerosas } \\
\hline Sim & 95 & 26,7 \\
\hline Não & 261 & 73,3 \\
\hline \multicolumn{3}{|l|}{ Mobilidade } \\
\hline \multicolumn{3}{|c|}{ Alcance, preensão e pinça } \\
\hline \multicolumn{3}{|c|}{ Incapaz de elevar os braços acima do nível do ombro } \\
\hline Sim & 21 & 5,9 \\
\hline Não & 335 & 94,1 \\
\hline \multicolumn{3}{|c|}{ Incapaz de manusear/segurar pequenos objetos } \\
\hline Sim & 13 & 3,7 \\
\hline Não & 343 & 96,3 \\
\hline
\end{tabular}

Fonte: dados da pesquisa. * IMC: Índice de Massa Corporal. ** CP: Circunferência da Panturrilha. 
Tabela 3. Continuação...

\begin{tabular}{|c|c|c|}
\hline Marcadores de Fragilidade & $\mathrm{n}$ & $\%$ \\
\hline \multicolumn{3}{|l|}{ Capacidade aeróbica e/ou muscular } \\
\hline \multicolumn{3}{|l|}{ Perda de peso não intencional } \\
\hline Sim & 38 & 10,7 \\
\hline Não & 318 & 89,3 \\
\hline \multicolumn{3}{|l|}{$\mathrm{IMC} *<22 \mathrm{Kg} / \mathrm{m}^{2}$} \\
\hline Sim & 66 & 18,5 \\
\hline Não & 290 & 81,5 \\
\hline \multicolumn{3}{|l|}{$\mathrm{CP} * *<31 \mathrm{~cm}$} \\
\hline Sim & 85 & 23,9 \\
\hline Não & 271 & 76,1 \\
\hline \multicolumn{3}{|l|}{ Teste de velocidade da marcha $>5 \mathrm{seg}$} \\
\hline Sim & 40 & 11,2 \\
\hline Não & 316 & 88,8 \\
\hline \multicolumn{3}{|l|}{ Marcha } \\
\hline \multicolumn{3}{|l|}{ Dificuldade para caminhar } \\
\hline Sim & 93 & 26,1 \\
\hline Não & 263 & 73,9 \\
\hline \multicolumn{3}{|l|}{$\geq 2$ quedas no último ano } \\
\hline $\operatorname{Sim}$ & 77 & 21,6 \\
\hline Não & 279 & 78,4 \\
\hline \multicolumn{3}{|l|}{ Continência esfincteriana } \\
\hline \multicolumn{3}{|l|}{ Perde urina ou fezes sem querer } \\
\hline Sim & 41 & 11,5 \\
\hline Não & 315 & 88,5 \\
\hline \multicolumn{3}{|l|}{ Comunicação } \\
\hline \multicolumn{3}{|l|}{ Visão } \\
\hline Sim & 150 & 42,1 \\
\hline Não & 206 & 57,9 \\
\hline \multicolumn{3}{|l|}{ Audição } \\
\hline Sim & 72 & 20,2 \\
\hline Não & 284 & 79,8 \\
\hline \multicolumn{3}{|l|}{ Comorbidades múltiplas } \\
\hline \multicolumn{3}{|l|}{ Polipatologia } \\
\hline Sim & 12 & 3,4 \\
\hline Não & 344 & 96,6 \\
\hline \multicolumn{3}{|l|}{ Polifarmácia } \\
\hline Sim & 56 & 15,7 \\
\hline Não & 300 & 84,3 \\
\hline \multicolumn{3}{|l|}{ Internação recente } \\
\hline Sim & 33 & 9,3 \\
\hline Não & 323 & 90,7 \\
\hline
\end{tabular}

Fonte: dados da pesquisa. * IMC: Índice de Massa Corporal. ** CP: Circunferência da Panturrilha.

processo de envelhecimento - como as doenças metabólicas e de curso crônico -, quais sejam: hipertensão arterial, diabetes mellitus tipo 2, câncer e doenças cardiovasculares. ${ }^{31}$

Outro importante marcador de fragilidade foi detectado em $23,6 \%$ dos participantes da pesquisa, os quais apresentavam a medida da panturrilha inferior a $31 \mathrm{~cm}$, demonstrando a presença de sarcopenia. Essa condição é caracterizada pela perda gradual e generalizada de massa, força e função muscular, acarretadas pela degeneração neuronal central e periférica, atrofia muscular e o aumento do tecido adiposo no músculo. A sarcopenia tem 
elevado prevalecimento e aumenta a ocorrência de incapacidades e dependência. ${ }^{32}$

A diminuição ou perda de massa muscular esquelética e consequente perda da função muscular associada ao envelhecimento, impactam negativamente as atividades de vida diária e aumenta a vulnerabilidade do idoso às quedas, culminando em dependência. ${ }^{33}$

No cenário internacional, os estudos voltados para a autopercepção da saúde são bastante empregados em pesquisas de base populacional. De modo similar, aqueles que evidenciam declínio na saúde e qualidade de vida dos idosos cresceram, determinando a autopercepção negativa da pessoa idosa. ${ }^{34}$

Os resultados mostraram que os idosos têm uma percepção ruim ou péssima da própria saúde, o que se torna um alerta quanto às repercussões desse potencial marcador para a fragilidade. Quando positiva, a autopercepção da saúde indica que o idoso possui autonomia, mobilidade e capacidade funcionais preservadas, assim como o desejo de manter-se ativo e independente em suas atividades diárias, condições importantes para o desenvolvimento de práticas preventivas de quedas. ${ }^{35}$

Outro estudo brasileiro encontrou forte associação entre a percepção ruim ou muito ruim do estado de saúde e a ocorrência de distúrbios crônicos de coluna, reforçando a autoavaliação da saúde pelo idoso como um indicador positivo da qualidade de vida e da morbidade. ${ }^{36}$ Apresentam duas vezes mais risco de mortalidade em cinco anos os indivíduos que autoavaliam a saúde como sendo negativa, se comparados com aqueles que a autoavaliam como excelente. ${ }^{37}$

De modo complementar, pesquisa nacional evidenciou elevada prevalência de autopercepção negativa do estado de saúde entre os idosos na faixa etária de 65 a 79 anos. Essa população apresentava fragilidade, sintomas depressivos e prestavam cuidados a alguém, sendo possível concluir que há uma forte associação desses fatores com a alta prevalência de autopercepção da saúde negativa. ${ }^{34}$ A elaboração de medidas pelos gestores de saúde, visando tornar a qualidade de vida da pessoa idosa melhor, podem ser subsidiadas por meio de estudos que abrangem as singularidades da autopercepção da saúde. ${ }^{38}$

Quanto à funcionalidade dos idosos, observou-se, na pesquisa, maior dependência para atividades instrumentais de vida diária. Tal efeito acontece porque a capacidade de realizar atividades complexas parece declinar de maneira mais rápida em relação às atividades básicas, o que pode, a curto prazo, aumentar o grau de dependência desses idosos. ${ }^{39}$ Assim, a expectativa é de que as AVD básicas não sejam as primeiras a serem afetadas no cotidiano dos idosos. Os prejuízos para realização de ambas as atividades representam perda de qualidade de vida para idosos, visto que, além de apresentar uma incapacidade funcional nas AVD instrumentais, pode ter relação com a autopercepção de saúde negativa. ${ }^{40}$

Por isso, é importante, na atenção à saúde, que a capacidade funcional do idoso seja avaliada, pois esta permite que os fatores de risco sejam identificados e que a evolução clínica dos problemas de saúde seja monitorada, como o surgimento de doença crônica ou suas complicações, risco de quedas, entre outros, possibilitando assim que a independência e autonomia do idoso sejam preservadas.

Outro fator potencial para a fragilidade, evidenciado nos resultados, foi a presença de idosos com perda de interesse em atividades antes prazerosas, e alterações de humor que sugestionam estados depressivos. As duas condições relacionam-se ao processo de dependência de AVD instrumental, uma vez que a depressão está associada à dependência e perda de autonomia. A depressão e a fragilidade podem ocorrer separadamente ou em conjunto. Um em cada dez idosos são frágeis ou apresentam sintomas depressivos, e uma alta porcentagem indica ocorrência das duas condições. ${ }^{41}$

A depressão precisa ser investigada em idosos, pois, se não identificada precocemente e o indivíduo não receber tratamento, é seguida pela fragilidade. Explica-se essa relação mediante mecanismos fisiológicos, porquanto as duas associam sintomatologia neural e condições como: fadiga, diminuição da velocidade da marcha, falta de atividade física, redução de massa corporal, morbidades, danos cognitivos e funcionais. ${ }^{42}$

Além dos marcadores de fragilidade discutidos, verificou-se a presença da polifarmácia. Resultado semelhante foi encontrado em pesquisa realizada com idosos institucionalizados na região Nordeste do Brasil, evidenciando que, devido à fragilidade, aumenta a necessidade e quantidade de medicamentos prescritos aos idosos. ${ }^{43}$

São indispensáveis e urgentes os programas de rastreamento e gestão da fragilidade nos ambientes de atendimento à saúde dos idosos. ${ }^{44}$ Por esse motivo, propõe-se que o rastreio da fragilidade seja incluído na avaliação multidimensional do idoso na Atenção Primária a Saúde (APS), objetivando a detecção dos marcadores de forma precoce. ${ }^{45}$

A APS representa um cenário oportuno e de grande importância para a enfermagem gerontológica na identificação precoce da fragilidade, pois é a porta de entrada do sistema de saúde responsável pelo acompanhamento próximo e longitudinal dos idosos. ${ }^{46} \mathrm{O}$ conhecimento do enfermeiro acerca da situação de saúde das pessoas idosas propicia o planejamento de ações efetivas de cuidado preventivo e reabilitador.

O aumento da expectativa de vida, o envelhecimento e a prevalência de doenças crônicas geram, no idoso, a dependência funcional. Portanto, é necessário capacitar os cuidadores, familiares e a equipe de saúde no reconhecimento precoce da fragilidade. ${ }^{47}$

Optou-se pelo uso do instrumento IVCF-20 para detecção da fragilidade, o qual é validado para o contexto brasileiro. ${ }^{48}$ Sua utilização mostrou-se apropriada no reconhecimento dos marcadores de fragilidade, sinalizando a sua importância em facilitar o rastreio de idosos frágeis pelo enfermeiro na APS. Nesse sentido, recomenda-se a integração deste instrumento para identificação e monitoramento da situação de saúde dos idosos na APS, com vistas ao aperfeiçoamento a assistência prestada ao idoso.

Não obstante, estudo apresenta algumas limitações a serem consideradas para a interpretação e generalização dos achados. O delineamento transversal impossibilita a determinação da casualidade; além disso, a amostra esteve limitada aos idosos assistidos por equipes da ESF de uma determinada cidade, 
indicando que os determinantes de fragilidade podem ser diferentes em outras regiões do país, e em outros níveis de atenção à saúde.

\section{CONCLUSÕES E IMPLICAÇÕES PARA A PRÁTICA}

Os marcadores de fragilidade apresentaram associação significativa com sexo, idade, estado civil, escolaridade, renda, presença de doença cardíaca e hipertensão arterial.

A identificação de idosos frágeis, noâmbito da Atenção Primária à Saúde, é fundamental para o planejamento das ações, subsidiando a prática assistencial do enfermeiro e demais profissionais da equipe que considerem as necessidades da pessoa idosa por meio da identificação precoce dos determinantes de fragilidade, condição preditora de incapacidades, institucionalização, hospitalização e morte.

O estudo, portanto, contribui na discussão e reflexão das ações à saúde da pessoa idosa, no tocante ao rastreio rápido dos fatores relacionados à fragilidade no âmbito da ESF.

\section{CONTRIBUIÇÕES DOS AUTORES}

Desenho do estudo: Ana Larissa Gomes Machado

Coleta ou produção dos dados; Pryscila Ravene Carvalho Oliveira, Vitória Eduarda Silva Rodrigues, Ana Karoline Lima de Oliveira, Francisco Gerlai Lima Oliveira, Gabriela Araújo Rocha

Análise de dados; Pryscila Ravene Carvalho Oliveira, Vitória Eduarda Silva Rodrigues, Gabriela Araújo Rocha, Ana Larissa Gomes Machado.

Interpretação dos resultados; Pryscila Ravene Carvalho Oliveira, Vitória Eduarda Silva Rodrigues, Ana Karoline Lima de Oliveira, Francisco Gerlai Lima Oliveira, Gabriela Araújo Rocha, Ana Larissa Gomes Machado.

Redação e revisão crítica do manuscrito; Pryscila Ravene Carvalho Oliveira, Vitória Eduarda Silva Rodrigues, Ana Karoline Lima de Oliveira, Francisco Gerlai Lima Oliveira, Gabriela Araújo Rocha, Ana Larissa Gomes Machado

Aprovação da versão final do artigo: Pryscila Ravene Carvalho Oliveira, Vitória Eduarda Silva Rodrigues, Ana Karoline Lima de Oliveira, Francisco Gerlai Lima Oliveira, Gabriela Araújo Rocha, Ana Larissa Gomes Machado

Responsabilidade por todos os aspectos do conteúdo e a integridade do artigo publicado: Pryscila Ravene Carvalho Oliveira, Vitória Eduarda Silva Rodrigues, Ana Karoline Lima de Oliveira, Francisco Gerlai Lima Oliveira, Gabriela Araújo Rocha, Ana Larissa Gomes Machado

\section{EDITOR ASSOCIADO}

Maria Catarina Salvador da Motta

\section{REFERÊNCIAS}

1. Alvarez ÂM, Sandri JVA. Population aging and the Nursing commitment. Rev Bras Enferm. 2018;71(Suppl 2):722-3. http://dx.doi.org/10.1590/00347167-201871sup201. PMid:29791632.
2. Sousa JAV, Lenardt MH, Grden CRB, Kusomota L, Dellaroza MSG, Betiolli SE. Physical frailty prediction model for the oldest old. Rev Lat Am Enfermagem. 2018;26(0):e3023. http://dx.doi.org/10.1590/15188345.2346.3023. PMid:30208156.

3. Alves EVC, Flesch LD, Cachioni M, Neri AL, Batistoni SST. A dupla vulnerabilidade de idosos cuidadores: Multimorbidade e sobrecarga percebida e suas associações com fragilidade. Rev Bras Geriatr Gerontol. 2018;21(3):312-22. http://dx.doi.org/10.1590/1981-22562018021.180050.

4. Carneiro JA, Ramos GCF, Barbosa ATF, Mendonça JMG, Costa FM, Caldeira AP. Prevalence and factors associated with frailty in noninstitutionalized older adults. Rev Bras Enferm. 2016;69(3):408-15 http://dx.doi.org/10.1590/0034-7167.2016690304i. PMid:27355291.

5. Fried LP, Tangen CM, Walston J, Newman AB, Hirsch C, Gottdiener $\mathrm{J}$ et al. Frailty in older adults: evidence for a phenotype. J Gerontol A Biol Sci Med Sci. 2001;56(3):146-56. http://dx.doi.org/10.1093/ gerona/56.3.M146. PMid:11253156.

6. Freire JCG, Nóbrega IRAP, Dutra MC, Silva LM, Duarte HA. Fatores associados à fragilidade em idosos hospitalizados: uma revisão integrativa. Saúde Debate. 2017;41(115):1199-211. http://dx.doi. org/10.1590/0103-1104201711517.

7. Faller JW, Pereira DDN, de Souza S, Nampo FK, Orlandi FS, Matumoto S. Instruments for the detection of frailty syndrome in older adults: a systematic review. PLoS One. 2019;14(4):e0216166. http://dx.doi. org/10.1371/journal.pone.0216166. PMid:31034516.

8. Sampaio LS, Carneiro JAO, Coqueiro RS, Fernandes MH. Indicadores antropométricos como preditores na determinação da fragilidade em idosos. Ciênc. saúde colet. 2017;22(12):4115-23. http://dx.doi. org/10.1590/1413-812320172212.05522016.

9. Lenardt MH, Carneiro NHK, Betiolli SE, Binotto MA, Ribeiro DKMN, Teixeira FFR. Fatores associados à força de preensão manual diminuída em idosos. Esc Anna Nery. 2016;20(4):e20160082. http://dx.doi. org/10.5935/1414-8145.20160082.

10. Bertolucci PHF, Brucki SMD, Campacci SR, Juliano Y. O Mini-Exame do Estado Mental em uma população geral: impacto da escolaridade. Arq. Neuro-Psiquiatr. 1994;52(1):1-7. http://dx.doi.org/10.1590/S0004282X1994000100001.

11. Ribeiro EG, Matozinhos FP, Guimarães GL, Couto AM, Azevedo RS Mendoza IYQ. Self perceived health and clinicalfunctional vulnerability of the elderly in Belo Horizonte/Minas Gerais. Rev Bras Enferm 2018;71(Suppl 2):860-7. http://dx.doi.org/10.1590/0034-7167-20170135. PMid:29791629.

12. Moraes EM, Lanna FM. Avaliação multidimensional do idoso [Internet]. 4 ed. Belo Horizonte: Editora Folium; 2014. [citado 2020 Jun 8]. (Coleção Guia de Bolso em Geriatria e Gerontologia, 1). Disponível em: https:// docplayer.com.br/14989491-Avaliacao-multidimensional-do-idoso.htm

13. Oliveira FGL, Rocha GA, Oliveira PRC, Rodrigues VES. Fatores associados à síndrome de fragilidade clínico funcional em idosos. In: Anais VI JOIN; 2019 Set 4-6, Salvador, BA. Campina Grande: Realize Editora; 2019.

14. Barreto MAM, Portes FA, Andrade L, Campos LB, Generoso FK. A feminização da velhice: uma abordagem biopsicossocial do fenômeno. Interfaces Científicas Humanas e Sociais. 2019;8(2):239-52. http:// dx.doi.org/10.17564/2316-3801.2019v8n2p239-252.

15. Sánchez-García S, Sánchez-Arenas R, García-Peña C, Rosas-Carrasco $O$, Avila-Funes JA, Ruiz-Arregui $L$ et al. Frailty among communitydwelling elderly Mexican people: prevalence and association with sociodemographic characteristics, health state and the use of health services. Geriatr Gerontol Int. 2014;14(2):395-402. http://dx.doi. org/10.1111/ggi.12114. PMid:23809887

16. Macklai NS, Spagnoli J, Junod J, Santos-Eggimann B. Prospective association of the SHARE operationalized frailty phenotype with adverse health outcomes: evidence from $60+$ community dwelling Europeans living in 11 countries. BMC Geriatr. 2013;13:3. http://dx.doi. org/10.1186/1471-2318-13-3. PMid:23286928.

17. Sousa NFS, Lima MG, Cesar CLG, Barros MBA. Envelhecimento ativo: prevalência e diferenças de gênero e idade em estudo de base populacional. Cad Saude Publica. 2018;34(11):e00173317. http:// dx.doi.org/10.1590/0102-311x00173317. PMid:30484561. 
18. Lins IL, Andrade LVR. Feminização da velhice: representação e silenciamento de demandas nos processos conferencistas de mulheres e pessoas idosas. Mediações. 2018;23(3):436-65. http://dx.doi. org/10.5433/2176-6665.2018v23n3p436.

19. Carneiro JA, Cardoso RR, Durães MS, Guedes MCA, Santos FL, Costa FM et al. Frailty in the elderly: prevalence and associated factors. Rev Bras Enferm. 2017;70(4):747-52. http://dx.doi.org/10.1590/0034-71672016-0633. PMid:28793104.

20. Rodrigues RAP, Fhon JRS, Pontes MLF, Silva AO, Haas VJ, Santos JLF. Frailty syndrome among elderly and associated factors: comparison of two cities. Rev Lat Am Enfermagem. 2018;26(0):e3100. http://dx.doi. org/10.1590/1518-8345.2897.3100. PMid:30517585.

21. Melo EMA, Marques APO, Leal MCC, Melo HMA. Síndrome de fragilidade e fatores associados em idosos residentes em instituições de longa permanência. Saúde Debate. 2018;42(117):468-80. http:// dx.doi.org/10.1590/0103-1104201811710.

22. Pimenta FB, Pinho L, Silveira MF, Botelho ACC. Fatores associados a doenças crônicas em idosos atendidos pela Estratégia de Saúde da Família. Ciênc. saúde colet. 2015;20(8):2489-98. http://dx.doi. org/10.1590/1413-81232015208.11742014

23. Melo BRS, Diniz MAA, Casemiro FG, Figueiredo LC, Santos-Orlandi $\mathrm{AA}$, Haas VJ et al. Avaliação cognitiva e funcional de idosos usuários do serviço público de saúde. Esc Anna Nery. 2017;21(4):1-8. http:// dx.doi.org/10.1590/2177-9465-ean-2016-0388.

24. Huguenin FM, Pinheiro RS, Almeida RMVR, Infantosi AFC. Caracterização dos padrões de variação dos cuidados de saúde a partir dos gastos com internações por infarto agudo do miocárdio no Sistema Único de Saúde. Rev Bras Epidemiol. 2016 jun;19(2):229-42. http://dx.doi. org/10.1590/1980-5497201600020002. PMid:27532748.

25. Fang JC, Ewald GA, Allen LA, Butler J, Westlake Canary CA, ColvinAdams $M$ et al. Advanced (stage D) heart failure: a statement from the Heart Failure Society of America Guidelines Committee. J Card Fail. 2015;21(6):519-34. http://dx.doi.org/10.1016/j.cardfail.2015.04.013. PMid:25953697.

26. Denfeld QE, Winters-Stone K, Mudd JO, Gelow JM, Kurdi S, Lee CS. The prevalence of frailty in heart failure: A systematic review and metaanalysis. Int J Cardiol. 2017;236:283-9. http://dx.doi.org/10.1016/j. ijcard.2017.01.153. PMid:28215466.

27. Pinheiro HA, Mucio AA, Oliveira LF. Prevalence and factors associated with the frailty syndrome in older adults in the Brazilian Federal District. Geriatr. Gerontol. Aging. 2020;14(1):8-14. http://dx.doi.org/10.5327/ Z2447-212320201900072.

28. Shinmura, K. Cardiac senescence, heart failure, and frailty: a triangle in elderly people. Keio J Med.2016;65(2):25-32. http://dx.doi.org/10.2302/ kjm.2015-0015-IR.

29. Farías-Antúnez S, Fassa AG. Frailty prevalence and associated factors in the elderly in Southern Brazil, 2014. Epidemiol Serv Saude. 2019;28(1):1-13. http://dx.doi.org/10.5123/S1679-49742019000100008. PMid:30916238.

30. Mello AC, Carvalho MS, Alves LC, Gomes VP, Engstrom EM. Consumo alimentar e antropometria relacionados à síndrome de fragilidade em idosos residentes em comunidade de baixa renda de um grande centro urbano. Cad Saude Publica. 2017;33(8):e00188815. http://dx.doi. org/10.1590/0102-311x00188815. PMid:28832786.

31. Santos RR, Bicalho MAC, Mota P, Oliveira DR, Moraes EN. Obesidade em idosos. Rev Med Minas Gerais. 2013;23(1):64-73. http://www.dx.doi. org/10.5935/2238-3182.20130011.

32. Diz JBM, Leopoldino OAA, Moreira BS, Henschk N, Dias RC, Pereira LSM et al. Prevalence of sarcopenia in older Brazilians: a systematic review and meta analysis. Geriatr Gerontol Int. 2017;17(1):5-16. http:// dx.doi.org/10.1111/ggi.12720.

33. Roh YH, Noh JH, Gong HS, Baek GH. Effect of low appendicular lean mass, grip strength, and gait speed on the functional outcome after surgery for distal radius fractures. Arch Osteoporos. 2017;12(1):41 http://dx.doi.org/10.1007/s11657-017-0335-2. PMid:28411349.

34. Carneiro JÁ, Gomes CAD, Durães W, Jesus DR, Chaves KLL, Lima $\mathrm{CA}$ et al. Autopercepção negativa da saúde: prevalência e fatores associados entre idosos assistidos em centro de referência. Ciênc. saúde colet. 2020;25(3):909-18. http://dx.doi.org/10.1590/141381232020253.16402018.

35. Gaspar ACM, Azevedo RCS, Reiners AAO, Mendes PA, Segri NJ. Fatores associados às práticas preventivas de quedas em idosos. Esc Anna Nery. 2017;21(2):e20170044. http://dx.doi.org/10.5935/14148145.20170044.

36. Romero DE, Santana D, Borges P, Marques A, Castanheira D, Rodrigues $\mathrm{JM}$ et al. Prevalência, fatores associados e limitações relacionados ao problema crônico de coluna entre adultos e idosos no Brasil. Cad Saude Publica. 2018;34(2):e00012817. http://dx.doi.org/10.1590/0102311x00012817. PMid:29513823

37. Reile R, Stickley A, Leinsalu M. Letter to the Editor of Public Health in response to 'Large variation in predictors of mortality by levels of selfrated health: results from an 18-year follow-up study'. Public Health. 2017;147:157-8. http://dx.doi.org/10.1016 / j.puhe.2017.03.017.

38. Medeiros SM, Silva LSR, Carneiro JA, Ramos GCF, Barbosa ATF, Caldeira AP. Fatores associados à autopercepção negativa da saúde entre idosos não institucionalizados de Montes Claros, Brasil. Ciên. saúde coletiva. 2016;21(11):3377-86. http://dx.doi.org/10.1590/1413812320152111.18752015.

39. Bendavan R, Cooper R, Wloch EG, Hofer SM, Piccinin AM, Muniz-Terrera G. Hierarchy and speed of loss in physical functioning: a comparison across older U.S. and english men and women. J Gerontol A Biol Sci Med Sci. 2017;72(8):1117-22. http://dx.doi.org/10.1093/gerona/glw209.

40. Sousa AA, Martins AM, Silveira MF, Coutinho WL, Freitas DA, Vasconcelos EL et al. Qualidade de vida e incapacidade funcional entre idosos cadastrados na estratégia de saúde da família. ABCS Health Sci. 2018;43(1):14-24. http://dx.doi.org/10.7322/abcshs.v43i1.986.

41. Vaughan L, Corbin AL, Goveas JS. Depression and frailty in later life: a systematic review. Clin Interv Aging. 2015;10:1947-58. http://dx.doi. org/10.2147/CIA.S69632. PMid:26719681.

42. Nascimento PP, Batistoni SS. Depressão e fragilidade na velhice: uma revisão narrativa das publicações de 2008-2018. Interface. 2019;23:e180609. http://dx.doi.org/10.1590/interface.180609.

43. Borges CL, Silva MJ, Clares JW, Bessa ME, Freitas MC. Avaliação da fragilidade de idosos institucionalizados. Acta Paul Enferm. 2013;26(4):31822. http://dx.doi.org/10.1590/S0103-21002013000400004.

44. Lenardt MH, Carneiro NH, Binotto MA, Willig MH, Lourenço TM, Albino J. Fragilidade e qualidade de vida de idosos usuários da atenção básica de saúde. Rev Bras Enferm. 2016;69(3):478-83. http://dx.doi. org/10.1590/0034-7167.2016690309i. PMid:27355296.

45. Brito KQ, Menezes TN, Olinda RA. Incapacidade funcional: condições de saúde e prática de atividade física em idosos. Rev Bras Enferm. 2016;69(5):825-32. http://dx.doi.org/10.1590/0034-7167.2016690502. PMid:27783723.

46. Lins ME, Marques AP, Leal MC, Barros RL. Risco de fragilidade em idosos comunitários assistidos na atenção básica de saúde e fatores associados. Saúde Debate. 2019;43(121):520-9. http://dx.doi. org/10.1590/0103-1104201912118.

47. Gavazzi G. Fragilité et vieillissement, concept et définitions. Rev Infirm. 2017;66(236):18-20. http://dx.doi.org/10.1016/j.revinf.2017.10.002. PMid:29179839.

48. Moraes EN, Carmo JA, Moraes FL, Azevedo RS, Machado CJ, Montilla DE. Índice de Vulnerabilidade Clínico Funcional-20 (IVCF20): reconhecimento rápido do idoso frágil. Rev Saude Publica. 2016;50:81. http://dx.doi.org/10.1590/s1518-8787.2016050006963. PMid:28099667. 